\title{
LAS POTENCIAS MEDIAS EN LA ARQUITECTURA CLIMÁTICA GLOBAL: LA HIBRIDACIÓN DE LA BRECHA NORTE- SUR
}

\section{María del Pilar Bueno ${ }^{1}$}

\section{Introducción}

La distribución del poder global ha sido uno de los debates más característicos de las Relaciones Internacionales. Unipolaridad, multipolaridad, bipolaridad y no polaridad son sólo algunos de los conceptos que promueven un análisis del Sistema Internacional y la vinculación entre actores estatales, particularmente Estados Nación. Desde finales del siglo XX, con el fin de la Guerra Fría y con ésta de la bipolaridad, quedaron de manifiesto el sinfín de desacuerdos académicos relativos a la existencia de un único o varios centros de poder en el escenario mundial. Sin embargo, la crisis de poder relativo que protagoniza la potencia hegemónica - Estados Unidos - acompañada por las dificultades que atraviesa Europa, ha colocado a las potencias medias en una esfera de privilegio.

Los BRICS - Brasil, Rusia, India, China y Sudáfrica - fueron así agrupados por Jim O’Neill, economista de Goldman Sachs. Su ensayo argumentaba que el potencial económico de estos países -excluyendo a

\footnotetext{
${ }^{1}$ Doctora en Relaciones Internacionales, Becaria Postdoctoral de CONICET, Docente de la Universidad Nacional de Rosario (UNR), Universidad Nacional de La Plata (UNLP)y de la Pontifica Universidad Católica Argentina (PUCA). Coordinadora del Departamento de Medio Ambiente del Instituto de Relaciones Internacionales de la UNLP, Directora de la Cátedra Latinoamericana de Ecología del Instituto de Cooperación Latinoamericana de la UNR y Coordinadora del Observatorio de Política Exterior Argentina de la UNR. E-mail: pilarbueno@hotmail.com.
} 
Sudáfrica que fue incorporado en 2011- los catapultaba para convertirse en economías dominantes para mediados del siglo XXI. La tesis no sólo acogió variables económicas sino demográficas, entre otros aspectos. Es por eso que se adujo que alcanzarían el $40 \%$ de la población mundial para la misma fecha mencionada.

La tesis BRICS no es la única que reconoce la creciente relevancia de un conjunto de actores que exceden a las potencias tradicionales o superpotencias. En tal sentido, las potencias medias han instaurado espacios propios, así como integran foros como resultado del reconocimiento externo o iniciativa de las potencias tradicionales. Dentro del primer grupo, puede mencionarse al Diálogo IBSA entre India, Brasil y Sudáfrica, el cual fue creado en el año 2003 a la luz del fracaso propiciado en las negociaciones comerciales de Cancún. Asimismo, el caso de BASIC que aglutina a países como Brasil, Sudáfrica, India y China en lo relativo al cambio climático, es un ejemplo válido del primer grupo. BASIC se convirtió en un actor climático relevante a partir de la quinceava Conferencia de las Partes (COP) de la Convención Marco de Naciones Unidas contra el Cambio Climático (CMNUCCC) celebrada en el año 2009 en la ciudad de Copenhague.

Como ejemplo del segundo grupo, es decir, de aquellos foros que han sido creados por actores externos a las potencias medias como reconocimiento de su creciente rol en el Sistema Internacional, es posible mencionar al Grupo de los 20. A partir de la crisis económica y financiera global, el G20 se convirtió en un espacio ampliado - ocupando así el lugar del G8 - de debate en materia económica. Allí fueron convocados países como Brasil, India, Sudáfrica, China, Argentina, Corea del Sur, Indonesia, México y Turquía.

Los debates acerca de la distribución de poder no sólo se limitan a la relación entre superpotencias y potencias medias, sino que también aluden a la tradicional división del mundo en países más y menos desarrollados o lo que se denomina la brecha Norte-Sur. Esta fisura ha caracterizado al Sistema Internacional desde el proceso de descolonización a partir del cual la arena internacional se vio azorada por el incremento numérico de los Estados. Este proceso tuvo hondas repercusiones en los foros internacionales, como por ejemplo Naciones Unidas, puesto que tal incremento significó el logro de mayorías en espacios donde cada Estado - no importando cuál sea - tiene un voto, como la Asamblea General. 


\section{María del Pilar Bueno}

El surgimiento y esplendor del debate relativo al telón que divide al mundo entre países industrializados y en desarrollo coincidió temporalmente con la inserción de los temas ambientales a la agenda global. En consecuencia, el nacimiento de estos tópicos estuvo plenamente ligado a dicha división. La cuestión climática no ha escapado a esta afirmación. Uno de los elementos más relevantes a la hora de caracterizar la brecha climática es el logro del Sur de que el Norte aceptara el principio de responsabilidades comunes pero diferenciadas, en la Cumbre de Río de Janeiro de 1992. Este principio se volvió un estandarte del mundo en desarrollo y fue plasmado en el Protocolo de Kyoto a través de la segmentación entre países del Anexo 1 y 2.

Uno de los espacios primordiales en los cuales se aglutinó el Sur para sostener sus posturas climáticas, fue el Grupo de los 77, el cual ha velado porque no se dé un paso atrás en las conquistas históricas. Si bien el G77 ha estado atravesado por subgrupos de negociación - países exportadores de petróleo, pequeños países insulares y grupo africano, entre otros -, en parte se han sostenido en base al sostenimiento de posiciones estructurales comunes como el principio de las responsabilidades.

A partir de esta breve caracterización de elementos contextuales que caracterizan el objeto de estudio, resulta relevante expresar el propósito de este artículo, así como las hipótesis de las cuales partimos. El objetivo de esta contribución es analizar en qué sentido el grupo BASIC puede modificar la arquitectura climática global, haciendo foco en la brecha Norte-Sur Climática y su persistencia como categoría analítica. En tal sentido hipotetizamos que el grupo BASIC tiende a hibridar la brecha Norte-Sur Climática, en virtud de la discordia que generan sus posturas en contraste con las del Sur nucleado en el G77.

Para hacer frente al propósito puesto de manifiesto, dividimos este trabajo en dos partes. El primer segmento realiza un breve desarrollo teórico en lo relativo a las diferencias conceptuales entre términos como potencia media, potencia emergente y potencia regional, entre otras nociones. Si bien el objeto de este trabajo no es teórico, consideramos que esta aclaración es necesaria para dar lugar al análisis del rol de las potencias medias. La segunda parte se dispone a analizar el rol de las potencias medias en la arquitectura global del cambio 
climático, tomando al BASIC como principal espacio de representación de tales Estados. Para ello se parte de una reflexión en cuanto a la categoría de Sur.

\section{Elementos conceptuales}

Como sucede con otros conceptos teóricos, no hay acuerdo entre los académicos respecto al uso de un único concepto que designe a las potencias medias, países emergentes o Estados con crecientes atributos de poder en el Sistema Internacional. Quizás la obra de Cartens Holbraad, quien suscribe la definición que aparece en una comunicación de las Naciones Unidas, sea una de las más representativas. En tal sentido afirma: "potencias medias son aquellas que, debido a sus dimensiones, sus recursos materiales, su voluntad y capacidad de aceptar responsabilidades, su influencia y su estabilidad están en vías de convertirse en grandes potencias". El autor incluyó dentro de esta categoría tanto a lo que denominó potencias medias superiores como a las menores que a su vez comprendían subdivisiones donde se hallaban las potencias regionales. (Holbraad 1989).

Esta definición es notable puesto que no solo muestra recursos materiales de poder sino elementos como la voluntad y capacidad para aceptar responsabilidades, lo cual ha sido objeto de críticas hacia países como China, a quien se le atribuye la escasa predisposición a aceptar este tipo de compromisos, mucho más en el aspecto climático. Por otra parte, la definición presenta una lógica de potencialidad, puesto que asume que estos países se hallan en vías a convertirse en grandes potencias. Lo que convierte al concepto de potencia media en una característica en tránsito o en mudanza.

A pesar de las diferencias conceptuales entre los autores, pareciera existir un relativo consenso respecto a que las potencias medias son un grupo de Estados que se encuentran estructuralmente por debajo de los países desarrollados o más industrializados, mientras que las potencias regionales pueden implicar Estados periféricos o semiperiféricos que sobresalen entre aquellos que se posicionan en el mundo en desarrollo en función de su posicionamiento geográfico. Por otro lado, el concepto de potencia regional aparece más asociado a momentos históricos propios de la Guerra Fría, como el proceso de descolonización y la distensión. 


\section{María del Pilar Bueno}

Robert Cox afirma por su parte, que las potencias medias pueden estar en un rango medio de capacidades materiales e incluso mantenerse en una posición intermedia en casos de conflicto. Sin embargo, considera que las potencias medias a diferencias de las regionales, tienen un rol secundario en las alianzas de tipo militares y en general no poseen un lugar en los bloques regionales (Cox 1996).

Otros autores que abordan conceptualmente este debate generan sus propias categorías como Jordi Palou quien desarrolla nociones escalonadas como la de superpotencias o potencias mundiales, grandes potencias, potencias medias, Estados pequeños o débiles y microestados (Palou 1993). Otros casos a mencionar son los José Miguel Insulza quien los denomina potencias intermedias (Insulza 1986), y Esther Barbé quien clasifica a las potencias en superpotencias, potencias hegemónicas, grandes potencias, potencias medias y potencias regionales. La autora afirma que las potencias medias son los países de tamaño grande o medio con una diplomacia activa en ciertas áreas que les suponen prestigio, tales como España, Italia, Brasil, Argentina, India, México y Nigeria. Por otro lado, las potencias regionales pueden solaparse con las potencias medias como categorías que se aplican a los Estados que por su peso demográfico, económico o militar y su política en un marco regional concreto, desempañan el papel de gran potencia en un ámbito geográfico estableciendo las reglas de juego (Barbé 1995).

Finalmente, tomamos el concepto de Clarisa Giaccaglia, quien define potencia media como: "aquella unidad política que, sobre la base de ciertas capacidades materiales, se auto-percibe y es percibida por otros Estados como diferente tanto de los Estados pequeños como de las grandes potencias" (Giaccaglia 2006, 65).

\section{Las potencias medias y arquitectura climática global}

No es la primera vez que un puñado de países con crecimiento económico sostenido y que provienen del llamado Tercer Mundo intenta minar los pilares del régimen de Bretton Woods. La década del sesenta y del setenta fueron testigos privilegiados de cómo la descolonización fue el puntapié para que los llamados países de la Periferia o del Tercer Mundo, "el Sur", utilizara su mayoría numérica en espacios de relativa igualdad como la Asamblea General 
de Naciones Unidas. Esto les permitió sacar ventaja de foros de debate como la Conferencia de Naciones Unidas sobre Comercio y Desarrollo (UNCTAD) y construir grupos de negociación y presión como el Grupo de los 77 (G77) y No Alineados (NOAL). Todos ellos subsumidos ante la realidad impuesta por el bipolarismo de la Guerra Fría.

Efectivamente, incluso en los momentos de declinación relativa de la potencia hegemónica (Estados Unidos) como en los setenta o en la actualidad, la cooperación Sur-Sur ha encontrado límites internos y externos. En el primer caso, como resultado de la propia insolvencia del Sur para reducir las diferencias y bregar en conjunto en un frente más amplio. En el segundo caso, es preciso reconocer que las potencias hegemónicas fueron efectivas en la búsqueda de desarticular estrategias del Sur, como por ejemplo la iniciativa del Club de Deudores de la década del ochenta. Ambos elementos, el interno y externo tuvieron un efecto perjudicial sobre las iniciativas de negociación conjuntas.

Algunos académicos plantean que la propia concepción del Sur o de países en desarrollo proviene de una lógica automarginativa, al punto que se trata más que de un elemento meramente discusivo, de una identidad colectiva no desmentida ni por éstos ni por las superpotencias. Justamente Adil Najam (2005) esboza que el concepto de Sur surge como una categoría de autoexclusión que es apuntalada con la de periferia. Esto es, una noción de marginación, ausencia de poder y privación de derechos económicos a hacer negocios, lo cual ha redundado en un sistema internacional ilegítimo.

En consecuencia, a la pregunta acerca de si la categoría de Sur y a la propia brecha Norte-Sur, puede aún considerarse una categoría analítica válida para describir a una porción de actores del sistema internacional los autores han contestado colocándose a favor o en contra alternativamente (Miller 1992; 1995; 1998; 2000; Williams 2005; Berger 2004; Bayar 1991; Kamrava 1993; 1995; Krasner 1989). Najam afirma que la resiliencia del Sur ha desmoronado las hipótesis sobre su defunción conceptual y es desde allí que nos posicionamos en este trabajo. Consideramos que el mero recurso de discutir su actualidad denota que se encuentra tan viva como hace cinco décadas. Lo cual no significa que sea inmutable en sus márgenes o fronteras.

La perspectiva autocompasiva tiene algo de cierto si se afirma con Alberto Van Klaveren $(2012,132)$ que no es una coincidencia que el principal aporte de los países latinoamericanos a las relaciones internacionales haya sido 


\section{María del Pilar Bueno}

el enfoque de la dependencia, a la cual podríamos agregar otras teorías del sur, como la teoría del desarrollo, la teoría de la autonomía de Juan Carlos Puig (1971; 1980; 1984) y la teoría de la viabilidad de Hélio Jaguaribe (1964; 1969; $1972 \mathrm{a} ; 1972 \mathrm{~b} ; 1977 ; 1982 ; 1992)$.

Aun reconociendo la autopercepción de los países en desarrollo desde una perspectiva de exclusión, es interesante analizar cómo las cuestiones ambientales han jugado en este proceso. Si bien en los sesenta y setenta del siglo XX, cuando el tópico ingresó a la agenda global, se trató de una lógica de imposición del Norte al Sur (Estrada Oyuela 2007; Bueno 2010), a partir de la inclusión efectiva del concepto de desarrollo - principal preocupación del Sur hubo un cambio en la posición de este espacio o bloque hacia los temas ambientales. La incorporación plena del desarrollo sustentable desde la Cumbre de Río en adelante mostró, por un lado, el logro de articular iniciativas conjuntas y disolver diferencias, lo cual se vio plasmado en un número de documentos $^{2}$. Por otro lado y en consecuencia, denotó cómo los países del Sur articulados pueden lograr que el Norte ceda en algunas de sus posiciones más certeras. Caso contrario, es difícil explicar la incorporación de un principio como el de responsabilidades comunes pero diferenciadas que luego alcanzó materialidad con el Protocolo de Kyoto a la CMNUCCC.

A este respecto, Najam (2005) califica la acción ambiental de los países del Sur como contestataria previo y durante la Conferencia de Medio Ambiente Humano celebrada en Estocolmo en 1972; participativa desde esta fecha hasta la Cumbre de Río y de involucramiento en tiempos posteriores.

\footnotetext{
${ }^{2}$ Los cinco documentos firmados en Río fueron el puntapié para la profundización de las temáticas y la búsqueda de posteriores medidas de implementación que en muchos casos no llegaron, como fracasos posteriores de los regímenes. La Convención Marco contra el Cambio Climático y el Convenio de Diversidad Biológica dieron lugar a la celebración periódica de reuniones o conferencias de las partes que sostienen el régimen -con sus más y menos-; la Declaración de Río elevó un conjunto de herramientas jurídicas a principios de Derecho Internacional Ambiental con el reconocimiento de algunos aspectos esenciales como conquistas del Sur, como por ejemplo el principio de responsabilidades comunes pero diferenciadas y el principio de precaución. Asimismo, la Agenda 21 fue el primer -y para algunos el único- plan con medidas concretas y enfocadas en la incorporación decisional de los denominados Grupos de Río. Finalmente, la Declaración de Bosques no logró convertirse en un régimen similar al del clima o al diversidad por la propia puja de países en desarrollo, como Brasil, que se resistieron a suscribir un acuerdo que socavara su soberanía, tal como ellos mismos lo comprendieron.
} 
Ahora bien, nos preguntamos en qué se diferencia esta etapa de reconocimiento de las potencias medias con los registrados en tiempos precedentes. Podríamos reformular la pregunta diciendo: ¿en qué se diferencian las potencias medias del siglo XX de las del siglo XXI? La gran mayoría de analistas dan cuenta del carácter efímero de las tesis relativas al poder de los emergentes y su capacidad para socavar el poder de las superpotencias. Algunos ejemplos encontramos al recorrer la historia de países como Japón o los denominados tigres asiáticos, incluso algunos países latinoamericanos. Japón fue hasta fines del siglo XX, uno de los candidatos más estables a convertirse en una superpotencia; sin embargo, factores como el estancamiento de su economía hacia el noventa esfumaron tal pronóstico. Algo similar ocurrió con los tigres asiáticos, quienes alcanzaron altos niveles de crecimiento económico, industrialización y atracción de inversión extranjera directa hasta la crisis que se sucedió hacia 1997.

Frente a esto, es dable cuestionar qué diferencia a los denominados BRICS como pasibles de generar cambios permanentes en la estructura del poder global de países identificados como potencias medias con anterioridad. Si bien la tesis BRICS sigue estando a prueba a los fines de este trabajo, nos preguntamos en qué medida el cambio climático puede convalidar o echar por tierra esta teoría y cómo se vincula esto con la tradicional brecha Norte-Sur ambiental y climática. El G77 puede servir a manera de ejemplo. Se trató de uno de los pilares del surgimiento y desarrollo del Sur como entidad internacional y en su búsqueda de negociación aglutinada en los setenta. Sin embargo, es necesario analizar cómo se ha comportado en materia climática.

Actualmente el grupo aglutina cerca de 130 naciones entre las cuales se encuentran países con realidades locuazmente divergentes. No sólo nos referimos a aspectos tradicionales como variables económicas y sociales, sino a las identidades que caracterizan a los mismos como producto de una historia colectiva. Es así que el G77 aúna -entre otros- a los países exportadores de petróleo, a las naciones africanas y a los pequeños países insulares. Todos ellos muestran posturas discordantes en lo relativo al cambio climático. Mientras que los pequeños países insulares buscan medidas urgentes que frenen lo predicho por el Panel Intergubernamental de Expertos contra el Cambio Climático (IPCC), puesto que algunas de sus naciones perecerán como producto del crecimiento de las aguas; las naciones petroleras propenden a ralentizar los 


\section{María del Pilar Bueno}

esfuerzos por modificar las matrices energéticas propias y globales que disminuyan la demanda de aquello que garantiza su fuente de subsistencia, el petróleo.

Ya que existen diversos grupos de negociación transversales al G77, es válido cuestionar en qué sentido las potencias medias pueden modificar el orden/desorden climático global. Habría que analizar para ello los espacios e instrumentos que emplean para ejercer incidencia en la estructura climática global reciente. Consecuentemente, nos focalizamos en el grupo BASIC.

El grupo BASIC se convirtió en un actor climático relevante a partir de la COP- 15 celebrada en la ciudad de Copenhague. Sin duda, se trataba del gran desafío tras la hoja de ruta trazada en Bali en 2007 para lograr el ansiado Kyoto 2.0. Luego de las expectativas internacionales en este encuentro, la COP mostró los reveses de un sistema de conferencias mustio. Presidentes y primeros ministros que se superpusieron en sus arribos y por ende no negociaron directamente y con un anfitrión ansioso por lograr un acuerdo que al menos debía encausar el debate hacia una solución tras el vencimiento del Protocolo de Kyoto en 2012, representan sólo dos de los muchos elementos que pueden mencionarse.

La pérdida de liderazgo de Europa -baluarte tradicional del régimen climático- y mismo de Estados Unidos, tuvo como contracara un activismo de los países del BASIC que lograron un documento que para algunos analistas "salvó la cumbre". Es así que Copenhague se volvió una bisagra climática en términos del protagonismo de las potencias medias. Algunos de los aspectos que convalidan esta afirmación son: la aceptación a adoptar compromisos voluntarios en materia de reducción de emisiones y concentración energética; el protagonismo de estos actores en detrimento de otros como Estados Unidos o los países de Europa; y finalmente el malestar del G77 por la autoexclusión de éstos y su consolidación como líderes climáticos.

Probablemente es por eso que los miembros del BASIC se esforzaron por aclarar de continuo ${ }^{3}$ que no se trataba de una ruptura con el G77, ni de una

\footnotetext{
${ }^{3}$ La referencia al G77 está presente en todas las declaraciones y comunicados conjuntos que han realizado los países del BASIC desde la primera reunión de noviembre de 2009 en Beijing, China. Hay un esfuerzo particular por destacar que se constituye en un espacio de cooperación y negociación dentro del Grupo de los 77 y no por fuera de éste.
} 
esfera de competencia, puesto que no es un grupo de decisión política como sí el primero. Lo cierto es que sus posiciones más recientes rompen con las tradicionalmente sostenidas por el G77, comenzando por los compromisos voluntarios de reducción de emisiones de gases de efecto invernadero. Empero, es innegable que la condición de éstos es muy distinta a la de gran parte de los miembros del G77.

Tal como esbozamos en párrafos previos, al interior de este grupo perviven casos como el de China junto: Etiopía, Guinea, Liberia, Madagascar, Mozambique, Níger, República Centroafricana, Ruanda, Somalia o Uganda que según el Banco Mundial presentan 0.1 toneladas métricas per cápita de emisiones de dióxido de carbono. El caso chino es notable porque si bien se asienta en el primer o segundo lugar - dependiendo del origen del cálculo - como emisor global de gases de efecto invernadero a la atmósfera (emisiones totales), no sucede lo mismo en el valor de emisiones per cápita. Esto sucede como producto de la abundante población china de más de 1300 millones de habitantes frente a una población global de casi 7.000 millones. Algo similar ocurre con India, no así con Sudáfrica donde el valor per cápita es tan abultado como el de emisiones totales.

Ahora bien, si el G77 está compuesto por una variedad de países con realidades discrepantes, ¿cómo podría argumentarse que el BASIC rompe los consensos climáticos recientes del mundo en desarrollo, siendo que hay otros grupos como la Alianza de pequeños Países Insulares (AOSIS), el grupo de los Países Menos Adelantados (PMA), el Grupo de Integridad Ambiental o la propia OPEP, con posiciones tan opuestas entre sí? Es posible que la respuesta radique en que el BASIC se ha transformado en un trampolín que para algunos autores da cuenta del poder de veto climático de sus miembros (Viola, Franchini y Ribeiro 2012).

Las negociaciones propiciadas desde la COP- 13 celebrada en Bali han venido en caída. Éstas muestran la dificultad de alcanzar un acuerdo post Kyoto. Dicho debate sólo comienza con la reticencia de involucramiento de Estados Unidos amparado bajo el argumento de que China no puede continuar escondiéndose detrás de su condición de país en desarrollo para no asumir compromisos obligatorios. Varios países que conforman la Unión Europea se mostraron muy cercanos a este argumento, puesto que la Unión no sólo se conforma de Estados como Alemania o Francia sino de otros como los antiguos 


\section{María del Pilar Bueno}

países de la órbita soviética que aún pugnan por su propio desarrollo y más en el marco de la crisis financiera global. De esta forma, estas naciones no están dispuestas a financiar las emisiones globales y crecientes de China o de India.

Por otra parte, nos cuestionamos cómo se interpretará en un nuevo acuerdo climático global el principio de responsabilidades comunes pero diferenciadas. Si será como fue en los noventa, de una forma estricta, o más bien ajustado a las realidades actuales en términos de cantidad y concentración de emisiones.

Analizando los comunicados y declaraciones conjuntas desde noviembre de 2009, así como la vinculación entre éstos y las reuniones COP celebradas, resulta una media de cuatro reuniones anuales como espacios de negociación, consulta y acuerdo hacia las conferencias de las partes.

En la II Reunión Ministerial celebrada en el mes de enero de 2010 en la ciudad de New Delhi ${ }^{4}$ y luego de Copenhague, el grupo se autodefinió como un foro de acciones cooperativas, de mitigación y adaptación al cambio climático que incluye el intercambio de información. Asimismo, plantearon la relevancia de los grupos de trabajo ad hoc establecidos tanto en el marco de CMNUCCC como del Protocolo de Kyoto. Otro elemento que aparece aquí y se reitera en posteriores comunicados fue la relevancia de la implementación y el financiamiento de REDD+, es decir, del Programa de Reducción de Emisiones de carbono causadas por la Deforestación y la Degradación de los Bosques.

Luego del gran impacto negociador del BASIC en Copenhague (COP15, 2009), las diferencias se profundizaron, primero en Cancún (COP- 16, 2010) y luego en Durban (COP- 17, 2011). Si bien habían acordado en New Delhi destinar fondos propios a sustentar medidas de mitigación de países menos aventajados, medida que se adicionó a los compromisos voluntarios de la COP15; los desacuerdos se hicieron presentes. Los cuatro países discurrieron en lo relativo a la naturaleza legal del acuerdo climático futuro. Mientras India y China se opusieron a un acuerdo legalmente obligatorio, Sudáfrica y Brasil propendieron a aceptarla. A pesar de estas cuestiones, el cuarteto siempre ha

\footnotetext{
${ }^{4}$ Documento disponible en:

http://www.chinafaqs.org/files/chinainfo/BASIC\%20Joint\%20Statement\%2024\%20January\%202010. pdf
} 
sostenido que la base de cualquier acuerdo debe ser la equidad y el principio de responsabilidades comunes pero diferenciadas.

Previo a la celebración de la COP-16 en Cancún, el BASIC se reunió en varias oportunidades. La III Reunión Ministerial fue realizada en abril de 2010 en la ciudad sudafricana de Cape Town ${ }^{5}$. Entre los aspectos a destacar de la declaración conjunta es posible señalar la importancia de sostener la hoja de Ruta de Bali y la doble vía allí propuesta. Esto implica un doble acuerdo: un acuerdo sobre cuantificación de emisiones en el marco de un segundo período de compromisos post-Kyoto y un acuerdo de compromiso de largo plazo. Algunas de las áreas prioritarias señaladas en el documento son: los mecanismos de fast start o rápido financiamiento valuados en 10 billones de dólares; la implementación y financiamiento de REDD+; la arquitectura tecnológica para el desarrollo y transferencia; la generación de un marco de adaptación y la creación de un plan de trabajo relativo a MRV (measurement, reporting and verification) por parte de los países desarrollados.

Asimismo y previo al encuentro de Cancún se desplegó la IV Reunión Ministerial de julio de 2010 en la ciudad de Río de Janeiro ${ }^{6}$. Allí se reiteró la importancia del mecanismo de rápido financiamiento; la equidad; la necesidad de alcanzar un segundo período de compromisos post-Kyoto y otros aspectos como la preeminencia de que el financiamiento por parte de los países desarrollados provenga de fondos públicos. Lentamente, los primeros elementos se han vuelto la base de todas las declaraciones conjuntas. De hecho en la V Reunión ministerial celebrada en octubre de 2010 en la ciudad china de Tianjin ${ }^{7}$, no notamos adiciones a los aspectos centrales de los comunicados anteriores.

Tras la COP- 16 se produjo la VI Reunión Ministerial en febrero de 2011 en New Delhi ${ }^{8}$. Allí se analizan los resultados de Cancún con miras a Durban. Uno de los puntos notables fue la propia redacción de los acuerdos de Cancún, que de ninguna forma debía suplir a la Hoja de Ruta de Bali, desde la

\footnotetext{
${ }^{5}$ Documento disponible en http://www.moef.nic.in/downloads/public-information/BASIC-statement.pdf

${ }^{6}$ Documento disponible en http:/www.itamaraty.gov.br/sala-de-imprensa/notas-a-imprensa/jointstatement-issued-at-the-conclusion-of-the-fourth-meeting-of-ministers-of-the-basic-group-rio-dejaneiro-25-26-july-2010

7 Documento disponible en http://moef.nic.in/downloads/public-information/Fifth-BASIC-MinisterialMeeting-on-Climate-Change.pdf

${ }^{8}$ Documento disponible en http://moef.nic.in/downloads/public-information/BASIC-Stat-6.pdf
} 


\section{María del Pilar Bueno}

perspectiva de BASIC. El tema saliente y novedoso de la declaración es: la cuestión de la comparabilidad internacional incluyendo la rendición de cuentas y acción de los países en desarrollo en relación a los desarrollados que deben comprometerse seriamente con la mitigación y el financiamiento de acciones acordadas.

Hay un cambio significativo en la VII Reunión Ministerial de mayo de 2011 en Zimbali ${ }^{9}$, Sudáfrica, que responde a las acciones unilaterales de la Unión Europea relativas a la inclusión de las emisiones de la aviación en el régimen de comercio de emisiones. Frente a esta decisión, China pidió a sus aerolíneas no participar de dicho mercado, mientras que India se opuso discursivamente. El BASIC afirmó en su comunicado de Zimbali que este tipo de acciones no contribuyen a la construcción de un régimen multilateral sólido, no respeta el principio de responsabilidades comunes pero diferenciadas, ni otros principios sostenidos por ellos como la equidad. Además, hicieron un fuerte hincapié en el balance entre las medidas de mitigación y adaptación, cuestión que se verá profundizada desde Zimbali hasta las reuniones más recientes. La VIII Reunión Ministerial celebrada en agosto de 2011 en la ciudad de Inhotim, Brasil ${ }^{10}$ reiteró estos aspectos así como enfatizó la extensión del mandato de Kyoto y el compromiso de los países Anexo 1, en vistas a la finalización del período establecido en el mencionado Protocolo.

El último encuentro que realizaron los países del BASIC de miras a la COP-17 en Durban, fue la IX Reunión Ministerial de noviembre de 2011 en Beijing, China ${ }^{11}$. Allí recordaron como principios fundamentales de negociación a la equidad, el principio de responsabilidades comunes pero diferenciadas, las responsabilidades históricas, la hoja de Bali y su propuesta de una doble vía de acuerdos. Tal como se dijo previamente, estos cuatro aspectos se volvieron el pilar de todas las declaraciones y comunicados conjuntos. Asimismo, la necesidad de establecer un segundo período de compromisos bajo el mandato de Kyoto donde los países industrializados adopten compromisos claros de

\footnotetext{
${ }^{9}$ Documento disponible en:

http://moef.nic.in/downloads/public-information/Joint\%20statement \%20BASIC\%2029th\%20May.pdf

${ }^{10}$ Documento disponible en:

http://www.za.boell.org/downloads/BASIC_Joint_Statement_Inhotim_final_version.pdf

${ }^{11}$ Disponible en: http://www.indianembassy.org.cn/newsDetails.aspx?NewsId=267
} 
reducción. En tal sentido, un nuevo elemento de esta declaración fue que el mantenimiento de los mecanismos flexibles para los países Anexo 1 en un segundo período de compromisos debe depender del establecimiento de compromisos de reducción. Asimismo, que los países desarrollados deben proveer a un fondo de 30 mil billones de dólares para el denominado fast start. Por otro lado, apareció con claridad y por primera vez que no debe haber un balance entre mitigación y adaptación, sino un desequilibrio a favor de la segunda por la vulnerabilidad de los países menos adelantados. Finalmente, India propuso la inclusión de aspectos como el comercio equitativo y la propiedad intelectual en la agenda de la COP-17.

Luego de sucedido el encuentro propiciado en Durban, los países del BASIC - y como gesto para con uno de sus miembros que fue el anfitrión - en la X Reunión Ministerial realizada en febrero de 2012 en New Delhi ${ }^{12}$ valoraran el documento denominado la Plataforma de Durban, particularmente en lo relativo a: la conformación de un fondo verde global; el comité de adaptación; el comité ejecutivo de tecnología y un centro de tecnología climática. Asimismo, acordaron en la necesidad de un segundo período de compromisos de Kyoto y la presentación para mayo de 2012 - por parte de los países Anexo 1 - de información de reducción de emisiones y la posibilidad de enmendar el Anexo B del protocolo en este camino. Se recordaron a su vez el tema del mantenimiento de los mecanismos de flexibilización supeditado a compromisos cuantificables de reducción de los países Anexo 1. Finalmente, lamentaron el anuncio de Canadá sobre abandonar el acuerdo.

En cuanto a la XI Reunión Ministerial en julio de 2012 en Johannesburgo $^{13}$, el BASIC dio su apoyo a los documentos logrados en la Cumbre Río+20 como una forma de expresar condescendencia con su anfitrión. Asimismo, y a partir de allí, trasunta un fuerte énfasis en la implementación. De la misma forma, se manifestó preocupación por la información expresada en los informes denominados QELRGs (reducción cuantificada de emisiones u objetivos de reducción) en cuanto al avance en materia de reducción de

12 Documento disponible en:

http://moef.nic.in/downloads/public-information/10th-BASIC-Meeting-Delhi-Joint-Statement.pdf

13 Documento disponible en:

http://www.info.gov.za/speech/DynamicAction?pageid=461\&sid=29011\&tid=76046 


\section{María del Pilar Bueno}

emisiones de los países Anexo 1 y se hizo mención nuevamente a la necesidad imperiosa de contar con reglas comunes que habiliten la comparabilidad internacional. Los temas que mencionaron como mal logrados en la conferencia fueron: propiedad intelectual, medidas unilaterales europeas y cuestión de la equidad.

Cercana a la celebración de la COP-18 en la ciudad de Doha, se realizó la XII Reunión Ministerial en septiembre de 2012 en Brasilia ${ }^{14}$. Un elemento distintivo fue que contaron con la participación de otros representantes, espacio que designado BASIC plus. En este caso asistieron Barbados, Algeria (como presidente del G77+ China), Qatar (como anfitrión de la próxima COP) y Argentina. Allí reafirmaron la necesidad de que en Doha se pautase el comienzo del segundo período de compromisos a comenzar en enero de 2013; que los países Anexo 1 debían presentar información contundente sobre reducción de emisiones; la relevancia de la implementación - esto incluye el financiamiento a largo plazo -; y finalmente, la importancia de materializar el Fondo Verde Climático en Doha. Igualmente, que la base del acuerdo 2020 debía ser lo convenido en Bali, Copenhague, Cancún y Durban.

El último encuentro previo a Doha fue la XIII Reunión Ministerial en noviembre de 2012 en Beijing ${ }^{15}$. Dado que también se acogió al mecanismo de Basic plus, acudieron representantes de Algeria (como presidente del G77+China), Fiji (como miembro de la AOSIS y futuro presidente del G77+China) y Qatar (anfitrión de la COP- 18) como observadores. En esta declaración no hay aspectos novedosos, exceptuando: la efectivización de aquella advertencia acerca de que los países desarrollados que no participen del segundo compromiso no serán parte del mecanismo de desarrollo limpio de Kyoto. Igualmente, mostraron consternación porque los esfuerzos de mitigación de los países menos adelantados parecen mayores que los de aquellos industrializados. Esto no debe significar una transferencia de compromisos ni una inversión del principio de responsabilidades. Además, en cuanto a las

\footnotetext{
14 Documento disponible en:

http://www.itamaraty.gov.br/sala-de-imprensa/notas-a-imprensa/declaracao-conjunta-proferida-naconclusao-da-xii-reuniao-ministerial-do-basic-sobre-mudanca-do-clima-brasilia-brasil-20-e-21-desetembro-2012

${ }^{15}$ Documento disponible en: http:/www.indianembassy.org.cn/newsDetails.aspx?NewsId=381
} 
medidas unilaterales europeas, repudiaron la intención de la Unión Europea de sólo pausar su implementación por un año.

La COP-18 celebrada en Doha mostró el lado más renuente de algunos Estados a comprometerse en la extensión de Kyoto luego de su anunciada caducidad en 2012. Si bien Doha estableció una prórroga que llega a 2020, las partes actuales de Kyoto no llegan al 15\% de las emisiones totales puesto que Rusia, Japón y Canadá decidieron retirarse del acuerdo. A esta posición se agregaron otros estados como Belarús y Ucrania reticentes con la decisión de prorrogar Kyoto.

El documento de la COP, denominado Puerta Climática de Doha, no sólo exhibe el principal problema de comprometer a escasos países y un porcentaje limitado de emisores (Unión Europea, Australia, Noruega, Croacia), sino que prorroga el compromiso de lograr un nuevo pacto mundial por el clima a 2015 sin tener un objetivo global de reducción, ni profundizar un aspecto central como es la financiación, lo cual se aplaza decisionalmente a 2013. En este último sentido, el documento intenta impulsar el Fondo Verde para el Clima e insta a un acuerdo en la Cumbre de Varsovia de 2013 relativo a la cooperación del orden de los 100.000 millones de dólares por parte de los países industrializados.

Ante lo sucedido en Doha, los países del BASIC - en su XIV Reunión Ministerial de febrero de 2013 en la ciudad india de Chennai ${ }^{16}$ - manifestaron su decepción con la falta de compromiso de los países desarrollados en cuanto al financiamiento y la mitigación, tanto como la necesidad de que los Estados industrializados que no formaban parte del acuerdo original de Kyoto y del Anexo I adquieran deberes de mitigación en el marco de la CMNUCCC en el segundo período de compromisos. Asimismo, y enfatizando la lectura que realizan estos países del principio de responsabilidades comunes pero diferenciadas, establecieron que en función de las emisiones históricas de gases de efecto invernadero, es necesario cumplir con lo establecido por el IPCC en la necesidad de que los países Anexo I reduzcan sus emisiones al menos en un 25 a un $40 \%$ por debajo de los niveles de 1990 para 2020. Reiteraron que los países

${ }^{16}$ Documento disponible en http://moef.nic.in/assets/XIV BASIC Joint Statement FINAL.pdf 


\section{María del Pilar Bueno}

en desarrollo y entre ellos el BASIC, ha venido realizando mayores esfuerzos que los Estados más industrializados en vías a lograr lo establecido por la CMNUCCC. Justamente a este respecto y en lo que es, desde nuestro punto de vista, uno de los aspectos más destacables de la declaración, afirmaron que el objetivo de la Plataforma de Durban era reforzar los esfuerzos de todas las partes, fortaleciendo el régimen multilateral basado en normas y asegurando la implementación completa, efectiva y sustentable de la Convención para 2020 y de ninguna manera su relectura, renegociación o reinterpretación. Finalmente, y como ha aparecido en todos los documentos del BASIC, los países sostuvieron la unidad del grupo en sí mismo y de éste con el G77 + China y su compromiso por fortalecerlo en el marco de la cooperación sur-sur.

Los resultados de las últimas reuniones COP resultan para algunos académicos (Hurrell y Sengupta 2012), una muestra de la incapacidad de los emergentes para sostener las ganancias históricas y su status preferencial. Para otros, los compromisos asumidos por el BASIC forman parte de los cambios del sistema internacional en su estructura y dinámica e incluso la inexorabilidad del advenimiento de una nueva economía con bajos niveles de carbono (Viola, Franchini y Ribeiro 2012).

Valorando entonces las condiciones estructurales del Sistema Internacional y su lógica de poder, podemos afirmar que la división Norte-Sur desde una perspectiva climática parece gozar de buena salud. Esto es, fundamentalmente, los países en desarrollo continúan sosteniendo una postura a favor de las responsabilidades comunes pero diferenciadas. En términos del Derecho Internacional Ambiental, este principio proviene del reconocimiento del principio de igualdad soberana que aparece en diversos instrumentos internacionales, como por ejemplo la Carta de las Naciones Unidas. Hay un reconocimiento jurídico por parte de los países más industrializados en términos de su mayor contribución a la degradación ambiental y la mayor presión ejercida sobre los recursos. Además, no es sólo esto, sino que al incumplir su responsabilidad -más profusa- de proteger al ambiente, quedan responsabilizados por desfavorecer las propias posibilidades de que los países en desarrollo alcancen un mejor nivel de vida ${ }^{17}$.

\footnotetext{
${ }^{17}$ Para ampliar, ver en Borrás Pentinat, 2004.
} 
El ejercicio comparativo entre los declaraciones y comunicados de los ministros del BASIC y los documentos nacidos en las reuniones COP arrojan una alteración en la influencia ejercida por los grupos que participan en las conferencias a favor del espacio que comparten las cuatro potencias medias. Esto significa que su poder de influencia ha crecido en menoscabo de las potencias tradicionales como Estados Unidos - que ha continuado reticente en materia de compromisos obligatorios de reducción - y de Europa, como histórico baluarte de los regímenes climáticos. A favor de nuestro argumento aportan: la participación activa de los cuatro países en las COP; su condicionamiento del contenido de los documentos finales; el hecho de que los desacuerdos internos no los han resquebrajado como grupo; que han podido hacer frente común en caso de sentirse amenazados externamente como ocurrió con las medidas unilaterales de la Unión Europea; que han comprendido que su construcción de poder debe realizarse -al menos simbólicamente- dentro del G77 + China.

Sin embargo, y a pesar de que el BASIC presuma y reitere su pertenencia al Grupo de los 77 + China, las potencias medias que lo conforman han tendido a: resquebrajar el bloque de los 77, en función de su propia interpretación del principio de responsabilidades comunes pero diferenciadas; tener posturas en algunos casos contradictorias con el resto de Estados del grupo; y presentar diferencias que se profundizan en términos de cantidad y concentración de emisiones como producto del crecimiento económico, sea o no distributivo -o concentrado- en términos sociales entre ellos y el resto de los países que componen en grupo. Asimismo, por el establecimiento de baluartes propios como producto de la asiduidad de encuentros, lo que ha perfeccionado los mecanismos de cooperación y negociación, esto es: el principio de la equidad; el segundo período de compromiso de Kyoto; la vigencia de la CMNUCCC y su liderazgo junto con el Protocolo de Kyoto como regímenes climáticos internacionales; el principio de responsabilidades comunes pero diferenciadas; y el principio de las responsabilidades históricas.

A la pregunta entonces acerca de cómo pueden las potencias medias modificar el orden climático global, la respuesta que otorgamos es que el cambio climático muestra la hibridación en la brecha tradicional Norte-Sur. Este espacio que se abre es para algunos un espacio intermedio, siendo que para otros, que argumentan el poder de veto climático de aquellos, se trataría de una 


\section{María del Pilar Bueno}

transformación ostensible en la estructura y dinámica del Sistema Internacional.

\section{Conclusiones}

Este artículo se ha propuesto analizar si el grupo BASIC transforma la arquitectura climática global, haciendo énfasis en la brecha Norte-Sur y su persistencia como categoría analítica. A tal respecto, hemos afirmado que una de las características más relevantes de las potencias medias climáticas nucleadas en el grupo BASIC es que a diferencia de otros países emergentes han mostrado condiciones para digitar el juego climático en momentos en el que el mismo estaba trabado, como fue el caso de Copenhague. Asimismo, han sido reconocidos en su relevancia por las superpotencias y potencias tradicionales en las mesas de negociación. La decisión de Brasil, Sudáfrica, India y China de adoptar compromisos voluntarios de reducción puede leerse de muchas formas, aunque dos de ellas aparecen más relevantes en nuestro análisis: que se constituye en una pérdida de autonomía del Sur y un paso hacia tras como lectura del principio de responsabilidades; o que se trata de una posición inevitable en virtud del rol que ocupen estos países en la nómina de principales emisores globales.

En cualquier de los casos, esto significa un punto de corte con la lectura más inflexible de dicho principio que ha tendido a sostener el G77 como forma de defender las conquistas históricas del Sur. A nuestro parecer, si bien estos cuatro países continuarán negociando en el G77, el BASIC constituye ese trampolín al cual hemos hecho referencia. Una plataforma conjunta que al igual que otras -BRICS, IBSA- emplean como forma de patrocinar su crecimiento individual como jugares de las grandes ligas globales o jugares centrales.

No dudamos de la persistencia en la brecha Norte-Sur climática, sino que descartamos su estanquidad. Al igual que otras categorías analíticas, es mutable. La hibridación de dicha fisura por las potencias medias es un ejemplo de cambio, donde los cuatro países han sabido aprovechar los espacios vacíos que dejaron potencias tradicionales como Estados Unidos y los países de Europa en términos climáticos, así como hacer valer sus crecientes atributos de poder material, atravesar las divergencias y divisiones del resto de los países del Sur y emplear la asociación como un medio para multiplicar sus esfuerzos. A estos 
elementos podemos adicionar la posibilidad de demostrar su compromiso, voluntad y capacidad para aceptar responsabilidades y su potencial para convertirse en grandes potencias (Holbraad 1989). 


\section{María del Pilar Bueno}

\section{REFERÊNCIAS}

Barbé, Esther. 1995. Relaciones Internacionales. Madrid: Editorial Tecnos.

Bayart, Jean-Francois. 1991. "Finishing with the Idea of the Third World: The Concept of Political Trajectory". In Rethinking Third World Politics, editado por James Manor, 51-71. Harlow: Longman.

Berger, Mark. 2004. "After the Third World? History, destiny and the fate of Third Worldism". Third World Quarterly, 25(1): 31-39.

Borrás Pentinat, Susana. 2004. "Análisis jurídico del principio de responsabilidades comunes, pero diferenciadas". Revista Seqüência, (49): 153-195.

Bueno, María del Pilar. 2010. De Estocolmo a La Haya. La desarticulación de las políticas ambientales en la Argentina. Rosario: UNR Editora.

Cox, Robert. 1996. Approaches to World Order. Cambridge: Cambridge University Press.

Estrada Oyuela, Raúl. 2007. "La preocupación política por el ambiente y la administración". Aportes para el estado y la administración gubernamental, 13(24).

Giaccaglia, Clarisa. 2006. "Pequeños gigantes del sistema internacional: un estudio sobre la naturaleza y las funciones de las potencias medias". Trabalho de Conclusão de Curso, Facultad de Ciencia Política y Relaciones Internacionales de la Universidad Nacional de Rosario.

Holbraad, Carsten. 1989. Las Potencias Medias en la Política Internacional. Ciudad de México: Fondo de Cultura Económica.

Hurrell, Andrew; and Sandeep Sengupta. 2012. "Emerging powers, NorthSouth Relations and Global Climate Politics". International Affairs, 88(3): 463-484.

Insulza, J.M. 1986. "El dilema de una potencia intermedia". Revista Nexos (102).

Jaguaribe, Hélio. 1964. Desarrollo Económico y Desarrollo Político. Buenos Aires: Eudeba.

. 1972a. "Causas del Subdesarrollo Latinoamericano". In La crisis del desarrollismo y la nueva dependencia, organizado por José Matos Mar. Buenos Aires: Amorrortu.

. 1972b. Sociedad, Cambio y Sistema Político. Buenos Aires: Paidós. 
. 1977. "Implicaciones políticas del desarrollo de Latinoamérica". In

Poder y Desarrollo en América Latina, organizado por Aldo Solari. Ciudad de México: FCE.

. 1982. "Hegemonía céntrica y autonomía periférica". In América Latina

y el Nuevo Orden Económico Internacional, organizado por Eduardo

Hill e Luciano Tomassini. Buenos Aires: Editorial de Belgrano.

. 1992. "Experiencias y perspectivas de desarrollo". In Coloquio de

Invierno II: Las Américas en el Horizonte del Cambio. Ciudad de México: UNAM-FCE.

Jaguaribe, Hélio et al. 1969. La dependencia político-económica de América Latina. Ciudad de México: Siglo XXI.

Kamrava, Mhran. 1993. "Conceptualizing third world: the state-society seesaw". Third World Quaterly 14(4): 703-716.

. 1995. "Political Culture and a New Definition of the Third World"

Third World Quaterly 16(4): 691-703.

Krasner, Stephen. 1989. Conflicto estructural: el Tercer Mundo contra el Liberalismo Global. Buenos Aires: Grupo Editor Latinoamericano.

Miller, Marian. 1992. “Addressing Resource Management Concerns: The Third

World in Global Environmental Politics". In Development and Democratization in the Third World:Myths, Hopes and Realities, edited by Kenneth Bauzon, 169-181. New York: Taylor and Francis.

. 1995. The Third World in Global Environmental Politics. Buckingham: Open University Press.

. 1998. "Reconfigured: Environmental Regimes and Third World States". In The Greening of Sovereignty in World Politics, edited by Karen Liftin, 173-192. Cambridge: MIT Press.

. 2000. "Third World States and Fluid Sovereignty: Development Options and the Politics of Sustainable Ocean Management". Ocean and Coastal management, 43(2-3): 235-253.

Najam, Adil. 2005. "Developing Countries and Global Environmental Governance: From Contestation to Participation to Engagement". International Environmental Agreements 5: 303-321.

Palou, Jordi. 1993. "El concepto de potencia media. Los casos de España y México". Revista Cidob d'Afers Internacionals 26. 


\section{María del Pilar Bueno}

Puig, Juan Carlos. 1971. "La vocación autonomista en América Latina: heterodoxia y secesionismo". Revista de Derecho Internacional y Ciencias Diplomáticas 39/40.

. 1980. Doctrinas internacionales y Autonomía Latinoamericana. Caracas:

Instituto de Altos Estudios de América Latina, Universidad Simón Bolívar.

(ed.). 1984. América Latina: Políticas Exteriores Comparadas, Tomos I y II. Buenos Aires: Grupo Editor Latinoamericano.

Van Klaveren, Alberto. 2012. "América Latina en un nuevo mundo". Revista CIDOB d'Afers Internacionals 100: 131-150.

Viola, Eduardo; Franchini, Matias; e Thaís Lemos Ribeiro. 2012. "Climate governance in an international system under conservative hegemony: the role of major powers". Revista Brasilera de Política Internacional, 55 (edição especial): 9-29.

Williams, Marc. 2005. "The Third World and Global Environmental Negotiations: Interests, Institutions and Ideas". Global Environmental Politics 5(3): 48-69. 


\section{RESUMO}

El objetivo de esta contribución es analizar en qué sentido el grupo BASIC modifica la arquitectura climática global, haciendo foco en la brecha Norte-Sur climática y su persistencia como categoría analítica. La hipótesis es que el grupo BASIC tiende a hibridar la brecha Norte-Sur climática, en virtud de la discordia que generan sus posturas con el Sur nucleado en el G77.

\section{PALAVRAS-CHAVE}

Potencias Medias; Cambio Climático; División Norte-Sur. 